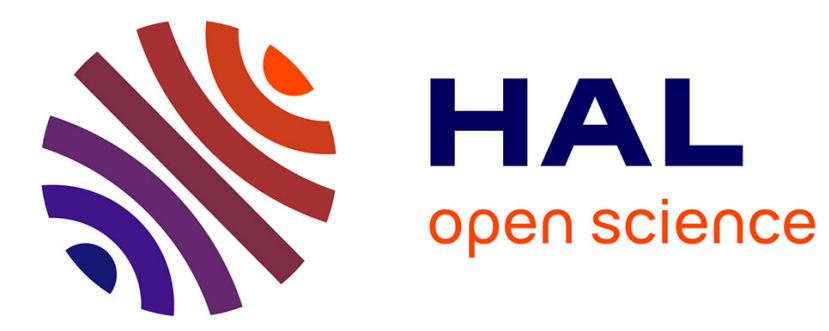

\title{
Mise en évidence des discontinuités spatiales et temporelles dans l'anthropisation de la plaine de la Vistrenque à Nîmes (Gard) durant l'Holocène. Apport de l'analyse malacologique
}

Sophie Martin, Frédéric Magnin, Pascale Chevillot

\section{To cite this version:}

Sophie Martin, Frédéric Magnin, Pascale Chevillot. Mise en évidence des discontinuités spatiales et temporelles dans l'anthropisation de la plaine de la Vistrenque à Nîmes (Gard) durant l'Holocène. Apport de l'analyse malacologique. Quaternaire, 2005, 16 (4), pp.339-353. halshs-00747923

\section{HAL Id: halshs-00747923 \\ https://shs.hal.science/halshs-00747923}

Submitted on 5 Nov 2012

HAL is a multi-disciplinary open access archive for the deposit and dissemination of scientific research documents, whether they are published or not. The documents may come from teaching and research institutions in France or abroad, or from public or private research centers.
L'archive ouverte pluridisciplinaire HAL, est destinée au dépôt et à la diffusion de documents scientifiques de niveau recherche, publiés ou non, émanant des établissements d'enseignement et de recherche français ou étrangers, des laboratoires publics ou privés. 


\title{
MISE EN ÉVIDENCE DES DISCONTINUITÉS SPATIALES ET TEMPORELLES DANS L'ANTHROPISATION DE LA PLAINE DE LA VISTRENQUE À NÎMES (GARD) DURANT L'HOLOCÈNE APPORT DE L'ANALYSE MALACOLOGIQUE
}

\author{
Sophie MARTIN*, Frédéric MAGNIN* et Pascale CHEVILLOT**
}

\begin{abstract}
RÉSUMÉ
La plaine de la Vistrenque à Nîmes (Gard) présente une occupation humaine extrêmement riche et ancienne, avec des structures archéologiques comprises au sein de séquences sédimentaires peu dilatées, très homogènes et tronquées. Malgré ces biais taphonomiques, une analyse malacologique a été effectuée afin de mettre en évidence l'évolution environnementale de la plaine en liaison avec l'anthropisation, à l'échelle d'un paysage. Les assemblages malacologiques montrent que la plaine de Nîmes subit très tôt, dès le début du Néolithique, un impact anthropique très fort. Un véritable agro-système néolithique se met en place et des paysages en mosaïque se maintiennent sur le long terme : ils comprennent des zones hautes dévolues à l'habitat et à l'agriculture (milieux rudéraux) et des zones basses dévouées au pastoralisme (prairies humides). Les seules phases de déprise agricole enregistrées interviennent aux périodes les plus récentes, Protohistoire et Antiquité. Pendant l'Antiquité, une gestion tout à fait originale de l'espace a permis localement une reconquête forestière. Enfin, l'artificialisation précoce des milieux a vraisemblablement eu pour conséquence le mauvais enregistrement de la reconquête forestière postglaciaire dans les séquences analysées.
\end{abstract}

Mots-clés : anthropisation, malacofaune, région méditerranéenne, archéologie, paléoenvironnement.

\section{ABSTRACT}

TEMPORAL AND SPACE DISCONTINUITIES OF THE HUMAN IMPACT IN THE VISTRENQUE BASIN (NÎMES, GARD) DURING THE HOLOCENE - CONTRIBUTION OF LAND SNAILS ANALYSIS

The Vistrenque plain in Nîmes (Gard, France) presents an old and extremely dense human occupation characterized by archaeological structures included in thin, homogeneous and truncated sedimentary sequences. In spite of these taphonomic biases, a malacological analysis was carried out in this area in order to highlight the environmental changes of the plain in relation to human impact on a landscape level. The land snail assemblages show that, as early as the beginning of Neolithic Period, the Nîmes plain is affected by a strong human impact. A true Neolithic agricultural system is then installed and mosaic landscapes are maintained on a long term: they include high zones devolved to habitat and agriculture (ruderal environment) and low zones devolved to grazing (wet meadows). The only phases of agricultural abandonment are situated during the most recent Periods, protohistorical Period and Roman Period. Roman Period corresponds to a completely original management of space, since a forest return is locally observed. Finally as a consequence of the early artificialisation of environment, the postglacial forest recovery had probably been badly recorded in the analyzed sequences.

Key-words: human impact, land snails, Mediterranean region, archaeology, palaeoenvironment.

\section{1 - INTRODUCTION}

Parce qu'elles répondent historiquement au souci d'élaborer des biozonations qui traduisent, pour l'essentiel, des variations climatiques, on a tendance à plutôt retenir des reconstitutions paléoenvironnementales les schémas généraux qu'elles proposent pour l'évolution du milieu. À l'Holocène, l'anthropisation du Midi de la France est ainsi souvent perçue, à l'échelle régionale, comme un phénomène caractérisé par une action humaine de plus en plus intense jusqu'au présent (par exemple, Vernet, 1991; Thiébault, 1995 ; Magnin, 1991 ; Triat-Laval, 1978), même si, localement et pour des périodes précises, des variations du degré d'anthropisation sont décrites (Berger et al., 2000). Finalement, ses modes et ses rythmes ont été peu étudiés en tant que tels, notamment par l'analyse malacologique, qui présente pourtant l'avantage de pouvoir reconstruire la structure de la végétation, et ses changements, à l'échelle locale (station) comme à l'échelle du paysage.

\footnotetext{
* Institut Méditerranéen d'Écologie et de Paléoécologie (IMEP - UMR 6116 CNRS), Université Aix-Marseille III, Bâtiment Villemin, Domaine du Petit Arbois, Avenue Philibert, BP 80, 13545 AIX EN PROVENCE CEDEX 04.

E-mails : sophie.martin@univ.u-3mrs.fr / frederic.magnin@univ.u-3mrs.fr

** CEREGE (UMR 6635 CNRS) / INRAP Méditerranée, 12 rue Régale, 30000 NÎMES. E-mail : pascalechevillot@wanadoo.fr
} 
En effet, les gastéropodes terrestres ont une résolution spatiale fine étant donné qu'ils sont fortement inféodés à leur habitat (Rousseau, 1985), qui, même lorsqu'il est de taille restreinte, peut permettre la survie de populations viables (Pfenninger, 2002). D'autre part, les malacofaunes sont particulièrement réactives aux perturbations qu'engendrent les différentes activités humaines sur l'environnement (Martin et al., 2003 ; Labaune et Magnin, 2002). Jusqu'aux périodes très récentes, ces activités concernent principalement des modifications de la structure de la végétation et de la structure des paysages, induites par le pastoralisme, l'agriculture et l'exploitation de la forêt. À une autre échelle, la distribution de certaines espèces et, par conséquent, la composition spécifique des peuplements malacologiques, sont dépendantes de la structure du paysage (Magnin et al., 1995 ; Magnin et Tatoni, 1995) et des flux de populations (humaines), d'animaux (troupeaux) ou de marchandises (semences, produits agricoles, ...) qui le traversent (Labaune, 2001). Il apparaît ainsi que l'analyse malacologique permet une bonne caractérisation de l'action de l'homme sur son milieu et de son évolution au cours du temps (Martin, 2004).

Les récents travaux d'aménagement de la plaine de la Vistrenque à Nîmes (Gard) ont permis de tester sur une fenêtre relativement large, dans un même contexte géomorphologique, climatique et biogéographique, la variabilité spatiale et temporelle de l'anthropisation de ce secteur du Languedoc oriental, à partir de l'étude des malacofaunes. La concentration des études paléoenvironnementales sur cette micro-région avait pour objectif de s'affranchir des reconstitutions trop stationnelles, ou a contrario trop générales, et de tenter de proposer une évolution à l'échelle d'un paysage.

Sept sites ont ainsi été analysés dans la partie sud-est de l'agglomération nîmoise, dans le cadre d'un vaste programme d'interventions d'archéologie préventive (SRA Languedoc-Roussillon, AFAN puis INRAP Méditerranée), soutenu par le Projet Collectif de Recherche «Espace rural et occupation du sol de la région nîmoise, de la Préhistoire à l'époque moderne » (coordonné par J.-Y. Breuil, INRAP).

\section{2 - AIRE D'ÉTUDE}

Les sept sites étudiés (Mas de Mayan, Mas de Vignole IV, Cadereau d'Alès, Fossé de Haute Magaille, Mas de Vignoles VI, Bassin Magaille Est, Mas de Vignoles VII) sont localisés dans la plaine de la Vistrenque, au sud-est de l'agglomération nîmoise (à moins de trois kilomètres du centre historique de Nîmes), à moins de deux kilomètres de la rive droite du cours d'eau actuel du Vistre (fig. 1).

La plaine alluviale du Vistre (la Vistrenque) forme une dépression étroite de 5 à $7 \mathrm{~km}$ de large qui sépare le domaine des Garrigues au nord, des Costières de Nîmes au sud. Le domaine des Garrigues est un paysage de collines et de plateaux d'altitude modeste (entre 100 et $200 \mathrm{~m}$ d'altitude), orientés selon un axe nord-est/sud-ouest. Il est formé de calcaires marneux du Crétacé inférieur (Darracq et al., 1984). Entre les Garrigues au nord et la plaine alluviale du Vistre, le piémont des collines calcaires du domaine des Garrigues correspond à un vaste coteau à pente faible qui s'est modelé en un glacis détritique au Pléistocène. Le domaine des Costières de Nîmes est, quant à lui, un ensemble de terrasses subhorizontales d'origine alluviale (d'une altitude maximale de $100 \mathrm{~m}$ ), façonnées par le Rhône au Quaternaire ancien et moyen.

La plaine de la Vistrenque a une altitude comprise entre 25 et $35 \mathrm{~m}$. Son substrat est composé de formations quaternaires, limons gris jaune calcaires d'origine éolienne et colluviale, reposant sur les cailloutis des Costières (non atteints dans les séquences sédimentaires étudiées). Ces formations superficielles ont été en partie remaniées à la fin du Tardiglaciaire et au début de l'Holocène par des phénomènes alluvio-colluviaux. Cette morphogenèse aboutit à la mise en place d'un modelé ondulant en talwegs et interfluves avec localement la formation de cônes ou nappes torrentiels plus ou moins étendus et coalescents (Jung et al., 2002), qui auraient été largement arasés par l'anthropisation dès le Haut Empire (Fabre et Monteil, 2001).

La Vistrenque est drainée par le petit fleuve méditerranéen du Vistre et toute une série de petits cours d'eau affluents issus des versants nord de la Costière et des versants sud des Garrigues (les cadereaux), qui incisent le piémont. Ils ont un régime d'écoulement torrentiel, actuellement temporaire (Fabre et Monteil, 2001); leurs débordements saisonniers, ainsi que ceux du Vistre, ont pu inonder une partie de la plaine et avoir également un rôle érosif. Certaines parties de la Vistrenque étaient encore marécageuses en 1948, avant le drainage et la canalisation du Vistre. Pour les périodes préhistoriques récentes, le régime hydrologique du Vistre et de l'ensemble de son réseau hydrographique a pu être différent, notamment influencé par un niveau marin situé plus bas qu'aujourd'hui (Breuil et Chevillot, 2000).

Les sites étudiés s'intègrent dans la zone de contact entre le piémont des Garrigues et la plaine du Vistre, où le complexe des formations du piémont s'imbrique plus ou moins dans les formations alluviales de la Vistrenque (Hervé, 2000a).

La zone d'étude, aux abords de Nîmes, présente une occupation dense et ancienne (fig. 2) avec notamment : des vestiges du Paléolithique supérieur, Magdalénien moyen ou supérieur ; une occupation épipaléolithique ; une occupation mésolithique, sauveterrienne; une occupation du Néolithique ancien épicardial ; des habitats du Néolithique moyen chasséen et du Néolithique récent de faciès Ferrières du Languedoc oriental; des grands habitats fossoyés du Chalcolithique, et plus précisément de faciès fontbuxien du Languedoc oriental et de faciès campaniforme ; des occupations protohistoriques (Bronze final, premier âge du Fer, second âge du Fer); des habitats et chemins antiques (Époque républicaine, Époque augustéenne, Haut Empire); des 

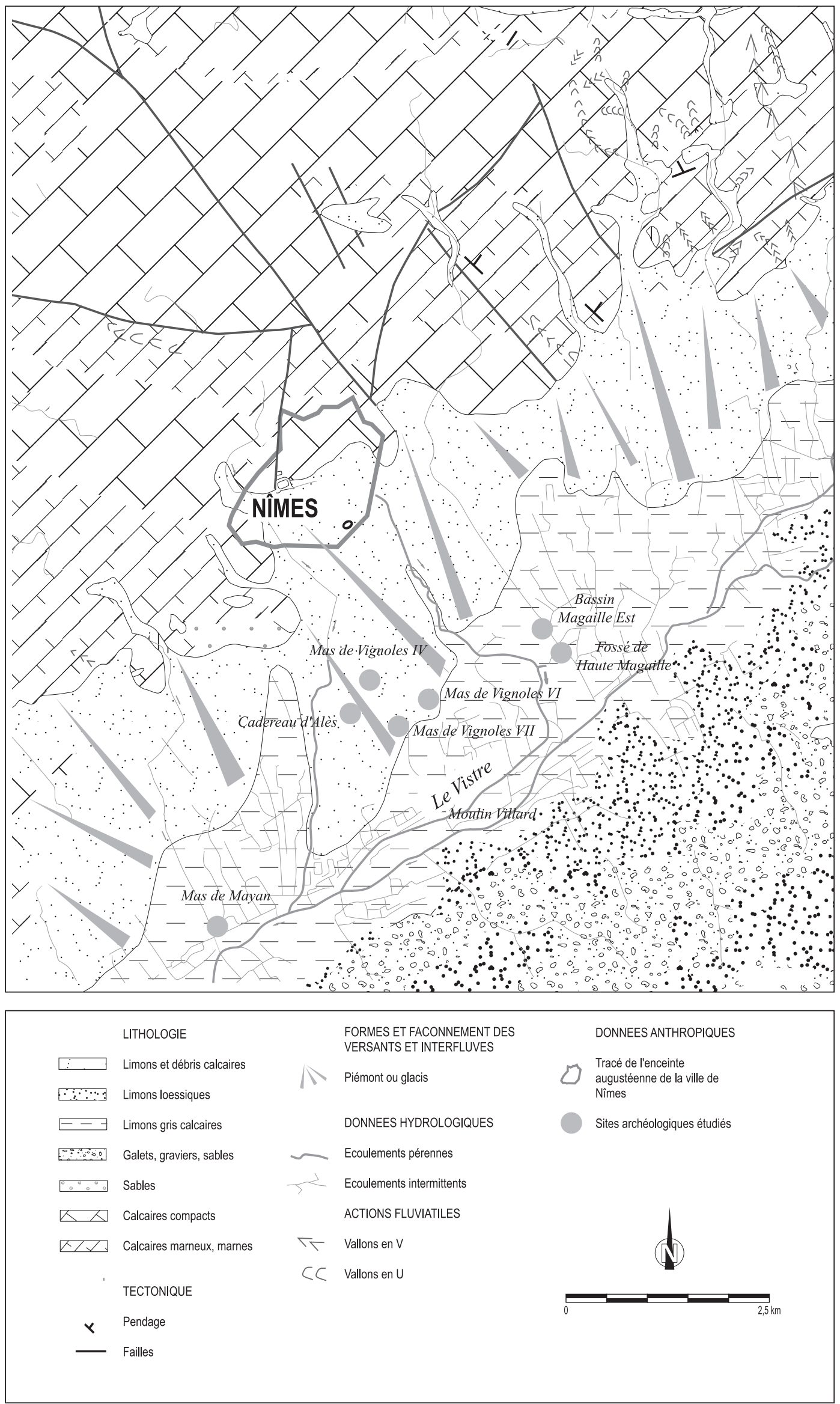

Fig. 1 : Croquis géomorphologique de la plaine nîmoise, d'après les cartes topographique et géologique de Nîmes (Gard) - DAO P. Chevillot et V. Lelièvre, INRAP.

Fig. 1: Geomorphological sketch of the Nîmes plain, according to the topographic and geological maps of Nîmes (Gard) - DAO P. Chevillot and V. Lelièvre, INRAP. 


\begin{tabular}{|c|c|c|c|c|}
\hline $\begin{array}{c}\text { cal BC } \\
\text { (d'aprés } \\
\text { Magny, 1995) }\end{array}$ & $\begin{array}{c}\text { BP (d'aprés } \\
\text { Magny, 1995) }\end{array}$ & $\begin{array}{c}\text { Chronozones } \\
\text { polliniques (d'aprés } \\
\text { Beaulieu (de) et } \\
\text { al., 1994) }\end{array}$ & Civilisations (d'aprés Jallot (ed.), 2005) & Sites analysés \\
\hline \multirow{4}{*}{820} & \multirow{4}{*}{2700} & SUBATLANTIQUE & $\begin{array}{l}\text { Epoque contemporaine } \\
\text { Epoque moderne }\end{array}$ & \multirow{5}{*}{$\begin{array}{l}\text { Bassin Magaille Est / Mas de Vignoles IV / Cadereau d'Alés / Fossé de Haute Magaille / Mas de Vignoles VII } \\
\text { Mas de Vignoles IV / Cadereau d'Alés / Bassin Magaille Est / Mas de Vignoles VII } \\
\text { |Mas de Vignoles IV } \\
\text { Mas de Vignoles IV }\end{array}$} \\
\hline & & & Moyen Âge & \\
\hline & & & \begin{tabular}{|l|} 
Antiquité \\
2 2nd Áge du Fer \\
1er Âge du Fer
\end{tabular} & \\
\hline & & \multirow[t]{2}{*}{ SUBBOREAL } & $\begin{array}{l}\text { Âge du Bronze final } \\
\text { Âge du Bronze moyen } \\
\text { Âge du Bronze ancien }\end{array}$ & \\
\hline 3450 & 4700 & & $\begin{array}{l}\text { Chalcolithique : Campaniforme / Fontbouisse } \\
\text { Néolithique final Ferriéres / Néolithique récent }\end{array}$ & \\
\hline 4880 & 6000 & ATLANTIQUE & $\begin{array}{l}\text { Néolithique moyen Chasséen } \\
\text { Néolithique ancien Epicardial } \\
\text { Néolithique ancien Cardial }\end{array}$ & Mas de Vignoles IV / Mas de Vignoles VI / Bassin Magaille Est / Mas de Vignoles VII \\
\hline 6900 & 8000 & BOREAL & \multirow[t]{2}{*}{ Mésolithique (Sauveterrien) } & \multirow[t]{2}{*}{ Mas de Vignoles VI } \\
\hline 8030 & 9000 & PREBOREAL & & \\
\hline 9200 & 10000 & DRYAS RECENT & \multirow[t]{2}{*}{ Epipaléolithique } & \multirow[t]{3}{*}{ Mas de Mayan } \\
\hline 10970 & 11000 & ALLERØD & & \\
\hline \multirow{2}{*}{13000} & \multirow{2}{*}{$\begin{array}{l}12000 \\
12700\end{array}$} & BÖLLING & & \\
\hline & & DRYAS ANCIEN & $\begin{array}{l}\text { Paléolithique supérieur (Magdalénien moyen et } \\
\text { supérieur) }\end{array}$ & Mas de Mayan / Cadereau d'Alés \\
\hline 16000 & 15000 & $\begin{array}{l}\text { PLENIGLACIAIRE } \\
\text { SUPERIEUR }\end{array}$ & & \\
\hline
\end{tabular}

Fig. 2 : Tableau chronologique et sites étudiés.

Fig. 2: Chronological table and studied sites.

réseaux complexes de fossés protohistoriques et antiques qui ont pu persister jusqu'à l'époque moderne voire contemporaine. Cette zone de la plaine du Vistre est comprise entre les axes nord-sud de deux voies antiques (Hervé, 2000b). L'aire d'étude est, ainsi, fortement soumise à l'influence de la ville antique de Nîmes et de l'agglomération protohistorique qui l'a précédée. L'absence remarquable de la longue période qui couvre le Bas Empire et tout le Moyen Ầge (Hervé, 2000b) est cependant à noter dans la zone d'étude.

\section{3 - LES SÉQUENCES SÉDIMENTAIRES ET LA STRATÉGIE D'ÉCHANTILLONNAGE}

Les structures archéologiques apparaissent globalement à faible profondeur, soit moins d'un mètre sous la surface actuelle du sol. Certaines, même, sont mises au jour juste sous les labours (Breuil et Chevillot, 2000 ; Pomarèdes et Rascalou, 2002).

On observe une extrême minceur des formations superficielles, notamment en ce qui concerne le début de l'Holocène, puisque les vestiges du Paléolithique supérieur ne sont découverts qu'à quelques centimètres sous les artéfacts néolithiques (site du Cadereau d'Alès par exemple). Ceci suggère la présence de lacunes sédimentaires, résultant de phases d'érosion. Nous reviendrons sur leurs possibles origines.

La stratigraphie est relativement homogène sur l'ensemble de la zone. Dans le détail, elle semble plus dilatée sur certains sites (Mas de Vignoles VI, Bassin Magaille Est, Mas de Vignoles VII) et relativement contractée sur d'autres points (Fossé de Haute
Magaille, Mas de Mayan, Mas de Vignoles VII, Cadereau d'Alès), en fonction d'une topographie ancienne régularisée par une morphogenèse très récente (fig. 3 ).

Elle comprend, de la base au sommet : (I) - Des colluvions jaune gris, interprétées comme un complexe lœssoïde pléistocène remanié, fortement carbonaté. Il présente des phénomènes d'oxydo-réduction liés aux fluctuations de la nappe phréatique. Ce sont à l'origine des dépôts de pente du piémont des Garrigues. Leur mise en place est due à l'érosion et au ruissellement sur le versant bordant la plaine. (II) - Un niveau brun jaune argilo-limoneux très riche en nodules de carbonates et en nodules de manganèse. Il s'agit de la partie sommitale, altérée, des dépôts lœssiques, caractérisée par une redistribution des carbonates (Breuil et Chevillot, 2000). Il daterait au moins du Tardiglaciaire. (IIIa) Une séquence limono-argileuse brun gris. La disposition des coquilles en différents horizons témoigne de la rythmicité de ces dépôts alluviaux (Chevillot in Piskorz et al., 2002). Elle est épaisse généralement de 0,20 à 0,40 m et elle correspond globalement à une accumulation néolithique puisque des occupations épicardiales ( site de Mas de Vignoles VI) sont observées à sa base et qu'on trouve des vestiges de l'âge du Bronze moyen/ancien (site de Mas de Vignoles VII) au toit de cette formation. Cette séquence hydromorphe prend place dans d'anciennes zones déprimées. En revanche, les zones hautes montrent, pour la même période, des niveaux sédimentaires différents constitués de sols calcaires colluviaux (IIIb). Cette répartition des profils de sols entre les zones basses et les zones hautes traduit des toposéquences. (IV) - Un niveau limoneux sableux compact brun clair ou gris jaune, très homogène, 


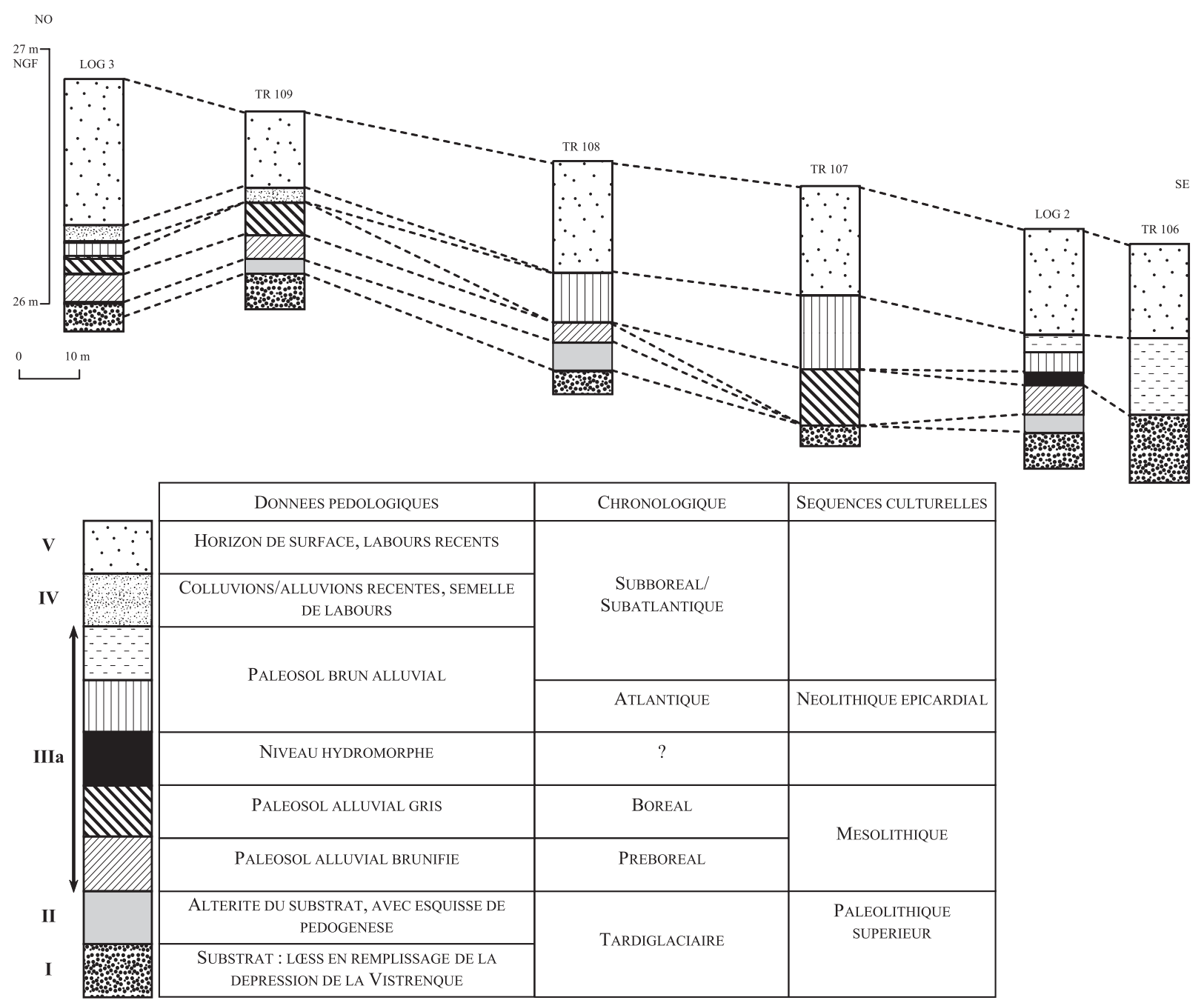

Fig. 3 : Profil stratigraphique du site de Mas de Vignoles VI (Nîmes, Gard), d'après Chevillot in Séjalon et al. (2004). Fig. 3: Stratigraphy of the site of Mas de Vignoles VI (Nîmes, Gard), according to Chevillot in Séjalon et al. (2004).

antique et post antique. (V) - Le niveau de labours comprenant du matériel céramique antique et moderne.

La principale difficulté des reconstructions paléoenvironnementales résulte du caractère lacunaire de la stratigraphie. Pour compléter l'étude des petites séquences sédimentaires, nous avons donc choisi d'intervenir également sur les structures archéologiques, dont le remplissage constituait un piège susceptible de suppléer aux lacunes de la stratigraphie globale. Afin de limiter le risque de n'enregistrer dans ces structures que les phases les plus drastiques de l'impact anthropique, les prélèvements ont été principalement faits sur les phases supérieures de comblement des structures, reflétant les périodes d'abandon de ces structures. C'est notamment le cas pour les ensembles de fosses néolithiques. Cependant, en fonction de la conservation des stratigraphies de comblement, certaines structures ont été intégralement étudiées (par exemple, le chemin antique ou les fossés néolithiques), afin de suivre l'évolution de l'impact anthropique au fur et à mesure de leur abandon.

\section{4 - MATÉRIEL ET MÉTHODE}

Sur les sept sites échantillonnés, 156 échantillons ont été prélevés au total. Ils consistent en des prélèvements de 10 litres de sédiment chacun, qui ont été séchés puis tamisés à l'eau sur un tamis à maille de $500 \mu \mathrm{m}$. Les refus ont été triés à l'aide d'une loupe binoculaire. Après extraction, les coquilles obtenues ont été déterminées et comptées, selon la méthode préconisée par Sparks (1961). L'identification des taxons et l'interprétation écologique ont été réalisées par comparaison avec une collection de référence (IMEP) et par le recours à des ouvrages de référence, notamment Kerney et Cameron (1999).

Ces données malacologiques sont présentées par un diagramme de synthèse réalisé grâce au logiciel Gpalwin (Goeury, 2000). La construction d'un tel diagramme regroupant les données de différents sites est méthodologiquement envisageable puisque ces sites sont disséminés dans une aire d'étude restreinte et homogène, présentant un même contexte climatique et géographique. 
Sur les 156 échantillons analysés, seuls 79 échantillons ont été sélectionnés pour l'élaboration de ce diagramme (fig. 4). Les échantillons dont la datation n'était pas très précise n'ont pas été pris en compte. Pour la période néolithique, qui montre une hydromorphie variable entre les zones déprimées et les zones plus hautes, seuls les échantillons correspondant aux zones hautes de la topographie ont été sélectionnés, pour des problèmes de représentation graphique. Notre commentaire prendra malgré tout en compte l'ensemble des données disponibles (zones hautes mais aussi zones basses). Au sein des grandes périodes individualisées (par exemple, l'âge du Fer), on a cherché autant que possible à ordonner les échantillons par ordre chronologique. Ce classement était impossible dans certains cas, par exemple pour certains échantillons fontbuxiens. En lisant le diagramme, il est donc préférable de prendre chaque période comme un tout au sein duquel l'ordre chronologique n'est pas toujours respecté.

Les échantillons sont datés par le matériel archéologique contenu dans le sédiment et par calage relatif entre les niveaux bien datés.

Pour notre propos, nous avons écarté les espèces aquatiques d'eau douce, qui ne renseignent pas directement sur les changements du couvert végétal mais plutôt sur les conditions de dépôt du sédiment. En tout, 90256 escargots participent à la construction du diagramme pour un total de 278261 escargots récoltés sur les 7 sites traités. Les espèces sont ordonnées sur le diagramme par affinité écologique et en fonction de notre connaissance des faunes méditerranéennes. Ainsi, Vallonia costata est placée parmi les espèces à affinité forestière : en effet, alors que cette espèce est présentée classiquement parmi les espèces de milieux ouverts (Kerney et Cameron, 1999), elle se retrouve fréquemment dans les litières forestières actuelles du domaine méditerranéen français (Magnin, 1991).

\section{5 - ASSEMBLAGES MALACOLOGIQUES ET PALÉOENVIRONNEMENT POSTGLACIAIRE}

\section{LE TARDIGLACIAIRE}

La base des séquences correspond à des niveaux tardiglaciaires lato sensu : en effet, même s'il n'y a pas d'éléments de datations plus précis, ces niveaux incluent, sur deux sites, des artefacts magdaléniens moyens et supérieurs. Les assemblages malacologiques indiquent la présence de milieux très ouverts, de type pelouse sèche (Candidula gigaxii, Candidula unifasciata). Ils comprennent encore quelques escargots de climat plus froid que l'actuel (Trochoidea geyeri). Ces assemblages comportent également parfois des éléments intrusifs, tels des Pomatias elegans ou des Cernuella virgata, au sein de niveaux probablement remaniés par des processus de bioturbations secondaires.
Dans certains secteurs, les assemblages analysés traduisent des milieux moins arides, de type prairies humides (Vallonia pulchella, Succinea oblonga). Un paysage en mosaïque est donc en place, probablement lié à une ancienne topographie, aujourd'hui gommée, la plaine du Vistre ayant été plus sensible aux crues de la rivière et de ses cadereaux ou aux remontées de nappe phréatique. C'est dans ce paysage hétérogène que s'installent les hommes du Magdalénien et de l'Épipaléolithique (site de Mas de Mayan), sans que la malacologie ne puisse enregistrer de variations locales de ce paysage pendant toute la période considérée, étant donné le probable brassage des différents horizons malacologiques.

Ces résultats sont conformes aux données anthracologiques (Heinz et Thiébault, 1998 ; Vernet, 1997) et palynologiques (Triat-Laval, 1978 ; Beaulieu (de) et $a l$, sous presse) régionales qui identifient la mise en place d'une «forêt-steppe » (pin sylvestre, genévrier, armoise, chénopodiacées), ouverte et sèche.

\section{L'HOLOCÈNE ANCIEN}

La première partie de l'Holocène est très mal représentée. Elle ne semble conservée que dans un site (Mas de Vignoles VI) correspondant à une ancienne zone déprimée, en liaison avec une occupation humaine sauveterrienne suivie d'une occupation épicardiale.

Les échantillons analysés sur ce site montrent que les espèces indiquant un climat plus froid que l'actuel ont effectivement disparu tandis que les espèces plus thermophiles continuent de se développer. Ces dernières traduisent la mise en place d'une couverture plus arborée (Pomatias elegans, Vallonia costata) qu'à la période précédente dans un contexte local très humide (Pupilla muscorum bigranata, Vertigo pygmaea, Vallonia pulchella), qui suggère donc le développement d'une ripisylve relativement ouverte.

Cette période correspond selon les données palynologiques et anthracologiques régionales à la mise en place de la chênaie caducifoliée (Triat-Laval, 1978 ; Vernet et Thiébault, 1987), qui caractérisera l'optimum climatique de l'Atlantique. L'établissement d'une ripisylve dans la plaine du Vistre pourrait être l'expression locale de l'extension des essences forestières mésophiles au début de l'Holocène. On observe cependant une dégradation de ce couvert arboré dès le Néolithique ancien épicardial (diminution des espèces à affinité forestière au profit des espèces de milieux ouverts).

\section{L'ATLANTIQUE RÉCENT ET LA PREMIËRE PARTIE DU SUBBORÉAL (NÉOLITHIQUE MOYEN, RÉCENT ET FINAL)}

À partir du Néolithique moyen chasséen, les assemblages de la Vistrenque traduisent une très forte diminution des espèces forestières, tandis que des espèces de pelouses sèches se développent considérablement (fig. 4). Le fait marquant de toute la période néolithique 


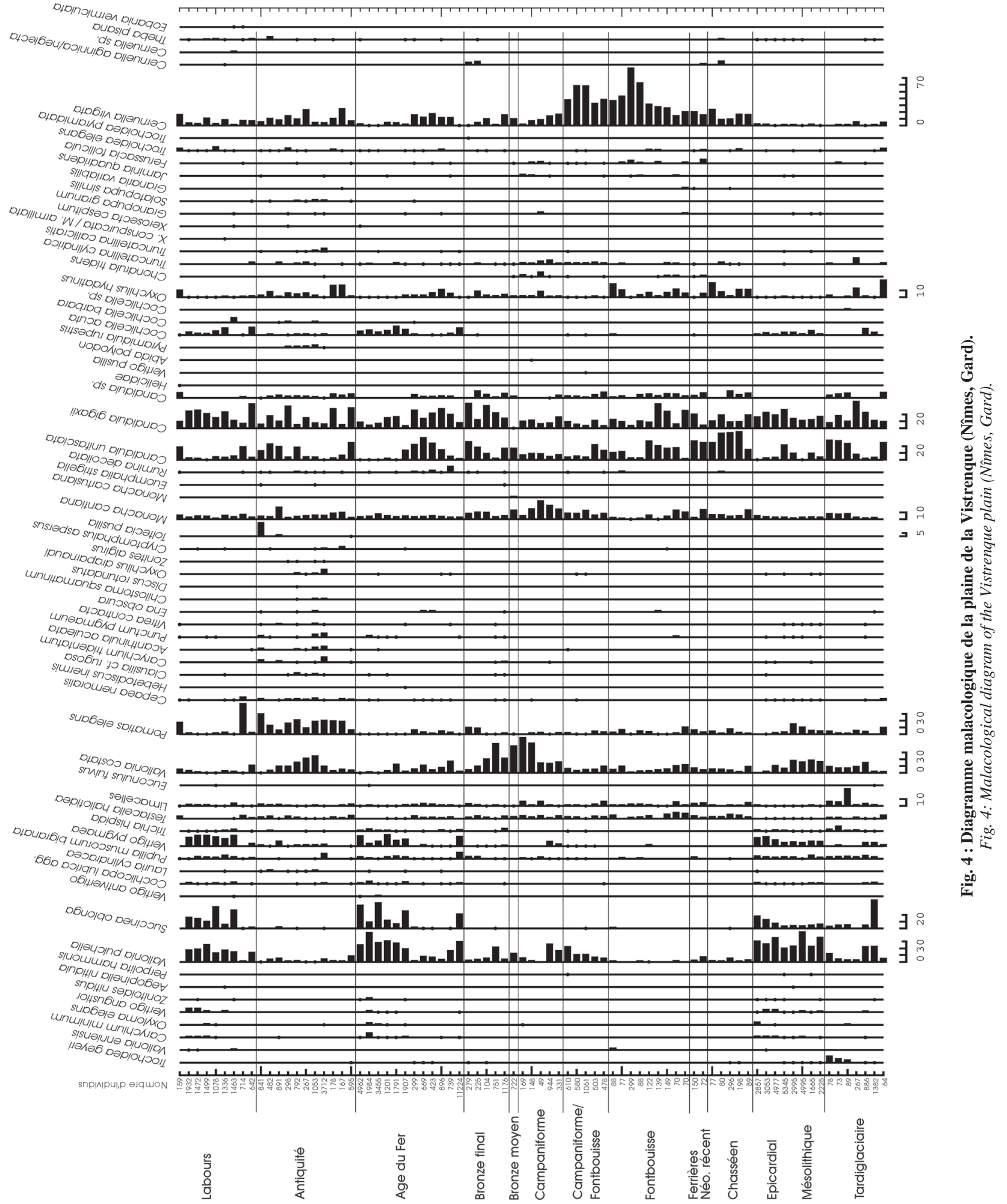


est l'apparition, à partir du Chasséen, de Cernuella virgata, une espèce caractéristique des milieux littoraux ou rudéraux, qui était totalement absente de la région avant cette période. Nous pouvons considérer ici que Cernuella virgata apparaît bien au Chasséen dans la plaine du Vistre : en effet, même si certains échantillons antérieurs peuvent contenir quelques individus de cette espèce, le contexte de prélèvement, la rareté de ces restes et l'observation macroscopique des sédiments nous suggèrent qu'il s'agit d'intrusions d'éléments plus récents dans des niveaux anciens.

Cernuella virgata est l'une des nombreuses espèces méditerranéennes apparues dans le Sud de la France au cours de l'Holocène. Elle était déjà présente au Néolithique ancien en Provence, au moins, sur le littoral (Magnin, 1991). Il semble que le facteur anthropique soit l'élément principal pour expliquer la dispersion, passive ou active, de cette espèce, grâce notamment à l'extension des milieux ouverts qui doivent avoir une certaine étendue pour assurer l'établissement de populations abondantes.

Ces assemblages malacologiques correspondent donc sans aucun doute à une anthropisation très forte de la plaine du Vistre dès le Chasséen, celle-ci s'accentuant encore au Néolithique final/Chalcolithique. En effet, d'une part, les populations de Cernuella virgata se développent rapidement au point que cette espèce devient prédominante au Néolithique final/Chalcolithique et, d'autre part, ces assemblages présentent une faible abondance, ce qui est un autre indice de la forte anthropisation du paysage (Boissinot et Magnin, 1992). Ainsi, si l'on peut considérer que des garrigues basses se maintiennent au Chasséen et au Néolithique récent, il semble que l'impact anthropique atteigne son paroxysme au Néolithique final fontbuxien, où le paysage est alors composé en grande partie de milieux herbacés plus ou moins rudéraux en relation avec la forte implantation de l'homme dans la plaine du Vistre.

La distinction, par la malacologie, entre les milieux typiquement rudéraux et les champs cultivés n'est pas évidente à établir, surtout s'il s'agit de cultures plus ou moins extensives ou temporaires. L'hypothèse de la pratique d'activités agricoles peut cependant être retenue ici étant donné que différentes études, soit archéologiques, soit palynologiques, ont établi cette pratique à proximité des sept sites étudiés, pour différentes périodes du Néolithique. Ainsi, au Chalcolithique, sur le site de Moulin Villard à Caissargues, la céréaliculture est attestée par la présence de pollens du type Cerealia (Planchais in Freitas (de) et al., 1987), associés aux taxons rudéraux. En outre, un champ cultivé du Chasséen est identifié par les archéologues sur les pentes du piémont des Garrigues dans la partie nord de l'agglomération nîmoise (Poupet et Jallot in Garmy et Monteil, 2000).

Ces milieux rudéraux sont observés dans les sites présentant des profils de sols calcaires colluviaux sur les anciens points hauts de la zone d'étude. Dans les zones basses ou très proches d'un cadereau latéral, les sols hydromorphes contiennent une malacofaune traduisant l'établissement de prairies humides (Vallonia pulchella, Succinea oblonga, Vertigo pygmaea), tandis que l'ancienne ripisylve a complètement disparu. Le maintien de prairies humides sur une longue période couvrant une grande partie du Néolithique, à partir du Chasséen, ne peut s'expliquer, selon nous, sans la permanence d'une anthropisation forte sur le paysage : en effet, dans le contexte climatique de l'optimum atlantique, ces formations prairiales devraient évoluer rapidement en formations plus fermées, au moins de type ripisylve; ici la pression de l'homme sur le milieu a nécessairement empêché la reconquête forestière. Au vu des assemblages présents, l'hypothèse d'une mise en pâturage des zones humides, basses ou proches des cadereaux latéraux, doit donc être envisagée.

Les résultats de l'analyse malacologique montrent ainsi la présence de paysages en mosaïque avec des milieux très ouverts juxtaposés à des prairies humides durant tout le Néolithique, du Chasséen jusqu' au Chalcolithique fontbuxien. Ces paysages sont marqués par un haut degré d' anthropisation ayant pour conséquence majeure l'absence complète de développement des milieux forestiers, au moins localement, que ce soit en contexte archéologique ou non.

Les études anthracologiques effectuées sur le site de Moulin Villard à Caissargues, situé sur la rive gauche du Vistre, en face des sept sites analysés dans cette étude (fig. 1), indiquent déjà une anthropisation du paysage au Fontbouisse, attestée par la prédominance des charbons de chêne vert (Blanchemanche et Chabal, 1995), qui s'accorde avec nos résultats et qui est également cohérente avec les analyses malacologiques effectuées pour ce même site (André in Freitas (de) et al., 1988). Par ailleurs, les résultats de l'anthracologie du Moulin Villard renforcent l'hypothèse proposée précédemment pour expliquer le maintien des formations de prairie humide. Nous avons effectivement suggéré qu'un pâturage des zones basses était possible. Or, Blanchemanche et Chabal (1995), toujours au Moulin Villard, indiquent que l'état déboisé de la plaine du Vistre au Chalcolithique (Fontbouisse) peut être attribué à l'élevage du bœuf et à l'existence de prairies en milieu défriché, étant donnée l'abondance d'ossements de bœuf sur le site. De plus, selon les archéologues spécialistes de la culture fontbuxienne, il semble que dans les zones de plaine, les groupes humains adoptent l'habitat fossoyé et vraisemblablement un élevage orienté vers l'exploitation préférentielle des bovins, tandis que l'élevage dans la garrigue semble plus orienté vers l'exploitation du cheptel ovin (Gutherz et Jallot, 1994).

C'est très nettement à partir du Campaniforme que l'on observe une diminution des espèces d'escargots de milieux rudéraux ou de pelouses (Cernuella virgata, Candidula sp.) au profit des espèces de broussailles et de prairies (Monacha cantiana, Vallonia pulchella, Vallonia costata). Ces assemblages malacologiques traduisent la mise en place dans les anciennes zones hautes de la plaine du Vistre d'un paysage à strate 
herbacée bien développée, soit de prairies assez denses soit de friches hautes. Ils correspondent donc à une diminution de l'impact anthropique sur le milieu et à une probable déprise agricole. La zone d'étude est cependant toujours occupée par les populations campaniformes, ce qui signifie que ce retour à des conditions plus fermées n'est pas dû à un abandon du site mais, plutôt, qu'il marque une mutation dans la gestion du paysage par rapport à la période précédente, et notamment le Néolithique fontbuxien. Ce changement des modes de gestion du paysage à la transition Fontbouisse/Campaniforme est d'autant plus intéressant qu'on peut l'observer, dans la continuité, sur des structures archéologiques qui ont servi aux deux groupes humains. Pour les zones hautes, nos observations suggèrent le passage d'une utilisation agricole ou pour l'habitat (Fontbouisse) à une utilisation probablement plus pastorale (Campaniforme). Ces hypothèses fondées sur l'analyse malacologique sont étayées par les résultats d'autres disciplines concernant un grand fossé campaniforme: l'étude archéozoologique indique une proportion tout à fait remarquable de restes de lapins et l'étude tracéologique de l'outillage lithique identifie un travail de coupe de végétaux « souples et tendres », différent des coupes de céréales (Convertini et al., 2004). Sans vouloir généraliser ces résultats ponctuels à l'ensemble de la culture campaniforme, on peut noter que cette gestion locale plus pastorale est en accord avec le modèle archéologique d'une économie campaniforme centrée sur les activités pastorales (Lemercier, 2000).

Enfin, si nous avons choisi de séparer chronologiquement les deux civilisations du Chalcolithique présentes dans la plaine du Vistre, le Fontbouisse et le Campaniforme, les études archéologiques récentes montrent qu'une contemporanéité de ces deux groupes est possible (Lemercier, 1998 ; Lemercier, 2000). Ainsi, plutôt qu'une évolution du paysage vers des milieux plus prairiaux au Campaniforme, on pourrait dans ce cas également interpréter nos données comme une juxtaposition, cette fois sur des espaces très réduits et dans les anciennes parties hautes de la Vistrenque, de différents types de milieux gérés différemment en fonction du groupe culturel qui les utilise.

\section{LA SECONDE PARTIE DU SUBBORÉAL (LA PROTOHISTOIRE)}

La reconquête végétale déjà notée pour le Campaniforme se poursuit à l'âge du Bronze : le milieu continue de se fermer progressivement avec une strate arbustive plus développée (Monacha cantiana, Vallonia costata ; quasi-disparition de Cernuella virgata). C'est l'âge du Bronze moyen qui correspond le mieux à cette tendance. Cependant les échantillons analysés pour l'âge du Bronze sont peu nombreux dans notre étude, et nous ne pouvons généraliser à l'ensemble de la zone étudiée l'image d'une relative déprise humaine à cette époque, même si du point de vue archéologique, la rareté des vestiges découverts sur l'ensemble de la commune de Nîmes (Monteil, 1999) s'accorde avec nos résultats.
Pour l'âge du Bronze final, les résultats anthracologiques du Moulin Villard à Caissargues donnent également l'image d'une reconquête forestière, avec la présence d'une forêt mésophile mixte, dite de plaine (frêne, chêne à feuillage caduc, orme) (Chabal, 1997). Ces résultats enregistrent la même tendance que nos données malacologiques : une déprise humaine a bien lieu à l'âge du Bronze, avec une remontée végétale dans la plaine. Les assemblages malacologiques du Bronze comportent cependant encore très peu d'espèces à affinité forestière. On observe ainsi un décalage entre les données paléoenvironnementales qui peut s'expliquer par des variations dans la résolution spatiale des assemblages malacologiques et anthracologiques. Il semble que la reconquête forestière mise en évidence par l'anthracologie ne soit pas généralisée à toute la plaine du Vistre et qu' un impact anthropique se maintienne sur la rive droite du Vistre, même s'il est moins fort qu'aux époques précédentes.

Le début de l'âge du Fer n'est pas représenté dans nos échantillons : en effet, les sites archéologiques sont pour l'instant très rares dans la plaine du Vistre, jusqu'au dernier quart du VI ${ }^{\mathrm{e}}$ siècle av. J.-C. (Monteil, 1999).

À partir de la toute fin du premier âge du Fer et pendant le second âge du Fer, les analyses malacologiques marquent une reprise d'un impact anthropique plus fort sur le paysage (diminution de Monacha cantiana et Vallonia costata; reprise de Cernuella virgata), mais qui reste sans comparaison avec le haut degré d'anthropisation identifié pour la période néolithique. À nouveau, un paysage en mosaïque est envisageable entre les zones hautes qui correspondent à des milieux de pelouse ou de garrigue basse (Candidula gigaxii, Candidula unifasciata) et les zones basses (ou proches d'un cadereau latéral) qui montrent des milieux de prairie humide (Cochlicella acuta, Vallonia pulchella, Succinea oblonga).

Ces résultats s'accordent avec les données palynologiques et archéologiques locales. En effet, les assemblages palynologiques du site de la ZAC des Halles, localisé à $2 \mathrm{~km}$ en amont des sites de la Vistrenque, traduisent, pour les $\mathrm{V}^{\mathrm{e}}$ et $\mathrm{II}^{\mathrm{e}}$ siècles av. J.-C., la présence de cultures (de blé et de seigle), mais également de zones de pâturage (Bui Thi Mai in Monteil, 1993). De plus, même si l'environnement est globalement très ouvert, il subsiste, d'après ces mêmes résultats palynologiques, un certain nombre d'essences forestières, tels les chênes caducifoliés et sempervirents (Bui Thi Mai in Monteil, 1993), ce qui pourrait également correspondre à l'image donnée par la malacologie d'une reprise d'un fort impact anthropique sur le site, cependant moins drastique qu'au Chalcolithique. Pour l'archéologie, le second âge du Fer correspond au développement de l'agglomération nîmoise préromaine, qui devient l'agglomération la plus importante du Languedoc oriental dès le courant du IV ${ }^{\mathrm{e}}$ siècle av. J.-C. (Monteil, 1999). Des champs labourés sont identifiés à Nîmes, dès la fin du IV ${ }^{\mathrm{e}}$ siècle av. J.-C., au Mont Cavalier, dans la partie nord de l'agglomération, et sur le site de la ZAC des Halles (Monteil, 1993 ; Garmy et 
Monteil, 2000). Le nombre de sites découverts pour cette période atteste que les campagnes protohistoriques étaient largement cultivées, structurées en quartiers et parcelles et parsemées de bâtiments (Garmy et Monteil, 2000), ce qui s'accorde avec nos données.

\section{LE SUBATLANTIQUE}

Les échantillons malacologiques de la période romaine (deuxième moitié du Ier siècle av. J.-C. jusqu'au III ${ }^{\mathrm{e}}$ siècle ap. J.-C.) montrent tous le développement des espèces forestières (Pomatias elegans, Acanthinula aculeata, Punctum pygmaeum, Vitrea contracta, Oxychilus draparnaudi, Toltecia pusilla) aux abords d'un chemin ou de fossés de parcellaire. Ils correspondent aux échantillons les plus forestiers, toutes périodes confondues, étudiés dans la plaine du Vistre. Ce sont principalement les échantillons du Haut Empire qui traduisent le mieux cette tendance. Ce développement des espèces forestières est, par ailleurs, moins nette à proximité de la petite ferme du site de Bassin Magaille Est.

Pour expliquer la présence de ces assemblages forestiers, on peut, dans un premier temps, suggérer l'existence de haies aux abords du chemin et des fossés de parcellaire antique. Cependant, les échantillons malacologiques antiques que nous avons étudiés comportent tout le cortège classique des espèces à affinité forestière qui se retrouvent dans les paysages actuels de forêts relativement ouvertes (Martin, 2004). Ils ne peuvent correspondre à l'établissement de haies bordières entre deux champs, très limitées spatialement. C'est pourquoi, il nous semble que de vrais milieux forestiers ont pu prendre place à l'époque antique. Par ailleurs, les échantillons analysés correspondent bien aux phases d'utilisation des structures (et non à une phase d'abandon) : en effet, pour le chemin antique de Vignoles, par exemple, plus les assemblages sont en contact avec les niveaux de circulation, et donc contemporains de l'utilisation, plus ils montrent des milieux fermés ; inversement, au fur et à mesure que les structures antiques sont abandonnées, on constate une réouverture des milieux sous l'influence d'une anthropisation postérieure aux phases d'utilisation des structures. Pour l'ensemble du paysage étudié, on peut donc imaginer un paysage en mosaïque avec, d'une part, des zones encore exploitées à proximité des fermes et, d'autre part, des zones où la reconquête forestière est effective.

Cette reconquête forestière n'est pas circonscrite à la plaine du Vistre puisque, sur le site de la ZAC des Halles, l'étude malacologique réalisée par Grenut et Peyric (Argant et al., 2001) montre également, pour la fin du Ier siècle ap. J.C. et le IIème siècle ap. J.-C., le développement de faunes sciaphiles et typiquement forestières, qui laissent percevoir un environnement où le couvert arboré s'est développé, alors que l'anthracologie identifie le retour de la chênaie verte sur le site à la même époque (Chabal in Monteil, 1993).
Cette reconquête forestière identifiée par la malacologie semble tout à fait étonnante pour l'époque romaine réputée correspondre à une extension des agrosystèmes. Cependant, du point de vue archéologique, les premiers signes d'une déprise urbaine sont perceptibles dès la fin du Ir siècle ap. J.-C. et le début du II ${ }^{e}$ siècle ap. J.-C. (Monteil, 1999), tandis que la périphérie du centre urbain est progressivement désertée (Garmy et Monteil, 2000). Ainsi, alors qu'une crise économique et/ou démographique est amorcée dès le ${ }^{\text {er }}$ siècle ap. J.-C., il est envisageable que le phénomène de déprise humaine ait aussi bien touché la campagne que la ville. Cette hypothèse a également été évoquée pour un autre site du Languedoc oriental, Lunel-Viel, qui connaît également une régénération des boisements et une certaine déprise agricole aux II ${ }^{\mathrm{e}}$ et III ${ }^{\mathrm{e}}$ siècles ap. J.-C. comme le montre l' analyse anthracologique (Chabal, 1997), alors que de nombreux sites ruraux sont abandonnés. Cependant, une deuxième hypothèse est possible pour expliquer ce paysage en mosaïque : les Gallo-romains ont pu également mettre en œuvre une gestion du paysage tout à fait originale par rapport aux périodes précédentes, dans laquelle des milieux très ouverts sont dévolus aux pratiques agricoles tandis que des milieux forestiers sont volontairement conservés et même entretenus.

Les données malacologiques concernant la période historique post-antique sont très disparates et ne sont pas très bien calées chronologiquement. La faiblesse de cet échantillonnage est liée à des séquences sédimentaires très contractées, associées à l'absence de sites archéologiques, qui attestent du faible taux de sédimentation et/ou de l'érosion anthropique des sols à la période historique. Malgré ces biais, les assemblages malacologiques semblent correspondre à un retour à des milieux très ouverts (Candidula gigaxii, Vallonia pulchella, Succinea oblonga et dans une moindre mesure Cernuella virgata), peut-être utilisés à des fins agricoles, dès le Haut Moyen Âge. Encore une fois, on observe un paysage en mosaïque, comprenant à la fois des milieux ouverts et secs et des prairies humides aux abords du Vistre, comme c'est également le cas sur le site voisin de la ZAC des Halles. En effet, les analyses malacologiques et palynologiques de ce dernier site témoignent de milieux très ouverts, rudéraux et cultivés en céréales, jouxtant des milieux pâturés (Argant et al., 2001).

Un échantillon moderne se distingue de cet ensemble post-antique par la forte prédominance des espèces forestières qu'il contient. Cette information ne peut cependant être généralisée à cause des attributions chronologiques assez floues. Il pourrait s'agir soit d'une reconquête forestière locale, soit d'une phase de diminution de l'emprise humaine sur le paysage.

\section{6 - DISCONTINUITÉS TAPHONOMIQUES ET ENVIRONNEMENTALES}

Ces résultats révèlent deux cas de discontinuités taphonomiques qui peuvent avoir des conséquences sur les reconstitutions environnementales que nous 
présentons : il s'agit de la rareté des échantillons de l'Holocène ancien et des périodes médiévales et modernes.

La rareté des échantillons de l'Holocène ancien dans les stratigraphies a pour principale conséquence que nous ne pouvons pas constater la mise en place de la chênaie caducifoliée de l'optimum atlantique, ni observer le moment où cette chênaie subit les premiers impacts anthropiques. Si le début de l'Holocène (au Boréal sur le site de Mas de Vignoles VI) se caractérise, dans la plaine du Vistre, par la mise en place de formations forestières encore très ouvertes, ce qui semble s'accorder avec les études paléoenvironnementales régionales, l'optimum de développement de la forêt à l'Atlantique ancien n'est pas visible dans le diagramme malacologique. Il y a deux raisons à cela. D'une part, on observe une dégradation locale des formations forestières dès l'Épicardial, qui ne peut être que la conséquence d'un impact anthropique précoce durant une période où la forêt est théoriquement en plein développement. D'autre part, on constate l'absence des niveaux correspondant à l'Atlantique ancien dans les autres séquences de la zone d'étude. Effectivement, des troncatures concernant la première partie de l'Holocène semblent assez systématiques dans les séquences sédimentaires de la plaine du Vistre. Ces troncatures ont pour conséquence l'absence d'information sur l'évolution environnementale des parties hautes de la zone d'étude avant les premiers niveaux chasséens. Or, au Chasséen, le paysage est déjà très largement ouvert et ne comporte plus de végétations arborées, même dans les contextes locaux de bord de cours d'eau, où la ripisylve a également disparu.

La rareté de ces niveaux stratigraphiques a également été observée par Triat-Laval (1978) pour la basse vallée du Rhône, avec notamment une lacune sédimentaire courante pour le Boréal. Berger et Brochier (2000) notent aussi l'occurrence d'une troncature généralisée, autour de $6000 \mathrm{BP}$, qui pourrait expliquer la plupart des lacunes sédimentaires de l'Holocène ancien en moyenne vallée du Rhône. L'Holocène ancien serait donc fréquemment éliminé des séquences sédimentaires de fonds de vallée du Sud de la France. Pour la plaine du Vistre, deux hypothèses peuvent être avancées pour expliquer l'érosion des sols et les troncatures dans les séquences : a) une reconquête forestière relativement lente qui induirait une faible couverture végétale inapte à jouer son rôle de protecteur des sols (Poupet in Garmy et Monteil, 2000 ; Jorda et Provansal, 1996) et qui donc ne suffirait pas à enrayer l'érosion dans le cas de phénomènes climatiques tels que les orages ; b) les conséquences d'impacts anthropiques d'envergure, comme l'érosion consécutive des premiers grands défrichements néolithiques. Si la deuxième hypothèse est parfois envisagée pour expliquer l'érosion des sols à partir du Néolithique moyen où elle est alors considérée comme le résultat «normal » du fonctionnement du géosystème méditerranéen anthropisé (Wainwright, 2000), elle est rarement retenue pour les périodes légèrement plus anciennes, qui s'insèrent pourtant dans des conditions climatiques dites optimales de l'Atlantique ancien (Magny, 1995). Une combinaison de ces deux hypothèses est largement envisageable pour expliquer cette troncature dans la plaine de la Vistrenque, d'autant plus que des impacts anthropiques d'importance sont détectés précocement dès l'Épicardial, et que, sur l'ensemble de la zone d'étude, le paysage est très largement ouvert au Chasséen. Ainsi, la présence de la forêt atlantique n'est pas visible dans la plaine du Vistre, soit parce qu'elle n'a jamais pu se mettre en place localement à cause d'une anthropisation forte dès l'Épicardial, soit parce que son développement a été stoppé par des défrichements d'importance qui s'expriment déjà fortement au Chasséen et qui ont pu conduire à l'érosion de ces horizons forestiers.

De même, les périodes historiques post-antiques se distinguent difficilement dans les séquences sédimentaires. La faible épaisseur de sédiment conservée après 1'Antiquité peut être due à des phénomènes d'érosion des sols, à mettre en liaison avec un labourage intensif de la zone. Les assemblages modernes, qui montrent des milieux très ouverts, peuvent être interprétés de deux façons : a) ils n'auraient enregistré que les phases ouvertes dans un ensemble plus varié, les sols « forestiers » ayant alors disparu des séquences. Dans ce caslà, l'unique échantillon moderne forestier pourrait constituer un piégeage de ces niveaux forestiers disparus. b) ils correspondent au maintien de milieux très ouverts pendant toute la période historique postantique. Les malacofaunes présentes dans ces niveaux ne traduiraient plus alors qu'un «empilement» de champs cultivés, dans un horizon cultural unique au sein duquel les assemblages malacologiques seraient mélangés, comme cela peut être le cas pour certains assemblages actuels ou subactuels (Martin et al., 2003). L'iconographie et les archives de la période moderne vont dans le sens de cette deuxième hypothèse, puisqu'elles montrent un paysage particulièrement dénudé sur les pentes du nord de l'agglomération nîmoise (Garmy et Monteil, 2000).

\section{7 - PRÉCOCITÉ ET CONTINUITÉ DE L'ANTHROPISATION À PARTIR DU DÉBUT DU NÉOLITHIQUE}

Le résultat principal de l'analyse malacologique de ce secteur de la plaine de la Vistrenque est l'extrême précocité et l'intensité de l'anthropisation à partir du début du Néolithique. Ce phénomène est d'autant plus marquant qu'il est relativement continu sur le long terme, sur l'ensemble du paysage. Les seules phases de relative déprise agricole correspondent aux périodes récentes, protohistoriques et antiques. Il semble donc que ce secteur de la plaine du Vistre ait connu, en plus d'une occupation humaine très dense dont témoigne l'abondance des structures archéologiques mises au jour, une utilisation intensive du paysage au Néolithique. 
Ce paysage ouvert s'est maintenu pendant tout le Néolithique moyen, récent et le Chalcolithique, ce qui implique une pérennité des pratiques agro-pastorales et le maintien d'un impact anthropique d'envergure sur la longue durée, fait tout à fait remarquable. Les archéologues ont montré que les plaines du Languedoc oriental sont densément peuplées au Chalcolithique (Gutherz et Jallot, 1994), vraisemblablement en liaison avec un essor démographique important (Lemercier, 1998), ce qui est tout à fait cohérent avec l'image d'un paysage très ouvert et très fortement anthropisé donné par la malacologie dans la Vistrenque. Cependant, cette forte anthropisation du paysage est encore plus intéressante pour le Chasséen, dont les sites retrouvés sont généralement moins nombreux.

Pour la région méditerranéenne française, ces résultats rejoignent ce qui a été observé dans la vallée du Lez (Hérault) : des formations de prairies humides prédominantes associées à des milieux plus secs et plus ouverts formant une juxtaposition de pratiques agropastorales au Néolithique (Martin et Magnin, sous presse ; Martin, 2004). Pour d'autres secteurs du Midi de la France, la dégradation des couverts forestiers au Néolithique est moins soutenue (Martin et Magnin, 2002 ; Martin, 2004) : des défrichements importants ont vraisemblablement lieu, mais ils ne sont pas rédhibitoires et sont probablement plus limités spatialement, contrairement à ce que l'on a pu observer pour la Vistrenque.

De manière générale cependant, les séquences analysées dans le Midi de la France, par la malacologie, traduisent toutes une ouverture des milieux dès le Néolithique (parfois cardial), les sites de la Vistrenque constituant le cas le plus remarquable. C'est un phénomène déjà bien connu par les études palynologiques et anthracologiques régionales (Triat-Laval, 1978 ; Puertas, 1998 ; Vernet et Thiébault, 1987 ; Vernet, 1997 ; Chabal, 1997). Cependant, le caractère ouvert des paysages néolithiques semble encore plus accentué à travers les analyses malacologiques. La résolution spatiale, relativement faible, des assemblages de gastéropodes terrestres et le fait qu'ils soient particulièrement performants pour reconstituer la structure des formations végétales permettent de penser qu'ils en donnent une image plus fidèle, sans pourtant, contrairement à l'anthracologie et à la palynologie, faire ressortir des schémas à signification spatiale plus large.

\section{8 - VARIABILITÉ SPATIALE DES MODES D'ANTHROPISATION}

L'analyse malacologique présentée ici met en avant des variations spatiales dans les modes d'anthropisation au cours de l'évolution de l'exploitation de la plaine du Vistre. C'est notamment le cas pour la période néolithique où la juxtaposition de deux types de milieux, l'un de prairies humides, l'autre de milieux rudéraux, indique l'établissement d'un paysage en mosaïque en fonction d'une gestion spatiale différente entre les zones hautes, liées à l'habitat et aux pratiques agricoles, et les zones basses (ou proches des cadereaux latéraux), probablement consacrées aux pratiques pastorales. Cette juxtaposition est le plus souvent parallèle à la mosaïque des sols liée à la topographie. Ces variations spatiales peuvent être comparées au schéma théorique que définit Wainwright (2000) pour un site rural, comprenant quatre zones présentant un degré d'anthropisation décroissant en fonction de l'éloignement à l'habitat : « le site même, qui est totalement anthropisé ; une zone agricole très anthropisée ; une zone pastorale moins anthropisée ; et une zone de marge peu ou non-anthropisée », en cercles concentriques. Même si ce schéma d'un « site catchment idéal » est défini pour l'âge du Bronze, nous pouvons le reprendre à notre compte pour les établissements néolithiques de la plaine du Vistre, avec, d'une part, le site d'habitat et sa zone agricole, dans les anciennes zones hautes de la plaine du Vistre, présentant des assemblages malacologiques de milieux rudéraux, et, d'autre part, la zone d'activité pastorale sur les parties plus déprimées et vraisemblablement plus proches du lit des anciens cours d'eau, présentant une malacofaune caractéristique des prairies humides. Enfin, la dernière zone, de marge, n'est pas mise en évidence par la malacologie, mais elle pourrait cependant correspondre à la chênaie verte identifiée par l'analyse anthracologique du Moulin Villard à Caissargues (en rive gauche du Vistre), au moins pour les niveaux chalcolithiques (Chabal, 1997). Ces résultats traduiraient l'établissement et la permanence d'un véritable système agro-pastoral dans la plaine du Vistre pour ces périodes anciennes.

La faible résolution spatiale des gastéropodes terrestres permet également d'identifier des gestions du paysage tout à fait originales, comme c'est le cas pour l'Antiquité. En effet, si, à l'échelle de la région méditerranéenne, cette période correspond à une ouverture drastique des milieux, provoquant souvent un effet de seuil déclenchant une crise environnementale (par exemple dans le Vaucluse, Martin et Magnin, 2002), en revanche, à Nîmes, les malacofaunes antiques récoltées traduisent incontestablement une reconquête forestière. Ainsi, alors que l'agglomération antique de Nîmes est l'une des plus dynamiques du Sud de la France, la malacologie met en évidence, au sein d'un paysage vraisemblablement largement exploité, des zones de la proche campagne où une déprise agricole est effective et où la pression humaine est en tout cas moins intense que pendant le Néolithique final/ Chalcolithique (Martin, 2004).

\section{9 - CONCLUSION}

Malgré un contexte sédimentaire peu favorable aux études paléoenvironnementales, les analyses malacologiques effectuées sur le secteur nîmois de la plaine de la Vistrenque ont mis en évidence la forte artificialisation 
des milieux dès le Néolithique ancien et un paysage largement modelé par l'homme dès le Néolithique moyen chasséen. Cette très forte anthropisation apparaît comme un phénomène continu pendant tout le Néolithique et s'exprime notamment par une gestion du paysage en mosaïque dans le cadre de la mise en place d'un véritable système agro-pastoral. Cette forte anthropisation pourrait également être la cause des importantes discontinuités sédimentaires constatées dans les séquences analysées. Le mauvais enregistrement de l'Holocène ancien trouverait ainsi son origine probable dans des crises environnementales liées à des impacts précoces et d'assez forte envergure au début du Néolithique, crises qui s'exprimeraient d'autant mieux qu'elles agissent sur des couvertures pédologiques et végétales encore fragiles. D'une façon encore plus drastique, l'intensification de l'anthropisation aux périodes médiévales et modernes induirait des érosions des sols, pouvant effacer d'éventuelles traces de déprises agricoles. Enfin, alors que le Néolithique final/Chalcolithique correspond à la période où l'impact humain est le plus fort dans la plaine de la Vistrenque, l'Antiquité, au contraire, enregistre localement, de manière originale, une reconquête forestière qui se produit en plein développement de l'agglomération de Nîmes, montrant ainsi la variabilité spatiale de la gestion des milieux.

\section{REMERCIEMENTS}

Nous remercions l'ensemble des participants au Projet Collectif de Recherche «Espace rural et occupation du sol de la région nîmoise, de la Préhistoire à l'époque moderne », et plus particulièrement son coordinateur Jean-Yves Breuil, ainsi que les archéologues avec qui nous avons travaillé sur les sites de la plaine du Vistre, Anne Hasler, Pierre Séjalon, Laurent Vidal, Luc Jallot et leurs équipes, pour leur précieux concours sur le terrain et les nombreux échanges scientifiques que nous avons eus ensemble. Nous remercions également Véronique Bonnet pour la relecture de l'abstract.

Nos remerciements vont enfin aux deux rapporteurs de cet article, Nicole Limondin et Jean-François Berger, pour l'ensemble de leurs remarques constructives.

\section{RÉFÉRENCES}

ARgant J., BARbERAN S., CHEVILlOT P., GRENUT I., MAUFRAS O., PEYRIC D., SELLAMI F., AMANDRY M. \& SAUVAGE L., 2001 - ZAC de la Gare II à Nîmes (Gard). Le paléochenal de la ZAC de la gare II à Nîmes : apport d'une fouille préventive dans la périphérie nîmoise à la connaissance de l'hydrologie et de l'environnement de la plaine du Vistre. Document final de synthèse, SRA Languedoc-Roussillon, INRAP, rapport non publié, Montpellier, $83 \mathrm{p}$.

BEAULIEU (DE) J.-L., RICHARD H., RUFFALDI P. \& CLERC J., 1994 - History of vegetation, climate and human action in the french Alps and the Jura over the last 15,000 years. Dissertationes Botanicae, 234, 253-275.

BEAULIEU (DE) J.-L., MIRAS Y., ANDRIEU V. \& GUITER F., sous presse - Vegetation dynamics in north-western Mediterranean regions: Instability of the Mediterranean bioclimate. Plant Biosystems.

BERGER J.F. \& BROCHIER J.L., 2000 - Évolution des paysages et des climats dans la moyenne vallée du Rhône et sa bordure préalpine de 13000 à $5000 \mathrm{BP}$. In « Les derniers chasseurs-cueilleurs d'Europe occidentale ». Actes du colloque international de Besançon, octobre 1998, Presses Universitaires Franc-Comtoises, Besançon, 37-57.
BERGER J.-F., MAGNIN F., THIEBAULT S. \& VITAL J., 2000 Emprise et déprise culturelle à l'Âge du Bronze : l'exemple du Bassin Valdainais (Drôme) et de la moyenne vallée du Rhône. Bulletin de la Société Préhistorique Française, 97 (1), 95-119.

BLANCHEMANCHE P. \& CHABAL L., 1995 - Potentialités forestières et activités humaines de la fin de la Préhistoire à la période historique dans le midi de la France : dégradation ou socialisation du milieu ? In S. Van der Leeuw (ed.), L'homme et la dégradation de l'environnement. $\mathrm{XV}^{\mathrm{e}}$ Rencontres Internationales d'Archéologie et d'Histoire d'Antibes, Éditions APDCA, Juanles-Pins, 207-229.

BOISSINOT P. \& MAGNIN F., 1992 - Le Verger 1: un site du $\mathrm{VI}^{\mathrm{e}}$ siècle av. J.-C. dans son environnement, au pied du Baou-Roux (Bouc-Bel-Air, B.-du-Rh.). Bulletin archéologique de Provence, 21, 15-30.

BREUIL J.-Y. \& CHEVILLOT P., 2000 - Bassin aval du Vistre de la Fontaine - Nîmes (Gard). Occupation néolithique et réseaux fossoyés antique et moderne. Document final de synthèse, SRA Languedoc-Roussillon, INRAP, rapport non publié, Montpellier, $110 \mathrm{p}$.

CHABAL L., 1997 - Forêts et sociétés en Languedoc (Néolithique final, Antiquité tardive). L'anthracologie, méthode et paléoécologie. Documents d'Archéologie Française, 63, Maison des Sciences de l'Homme, Paris, $189 \mathrm{p}$.

CONVERTINI F., FURESTIER R., ASTRUC L., FOREST V. \& JALLOT L., 2004 - Le Mas de Vignoles IV à Nîmes (Gard) : résultats préliminaires des fouilles du fossé à occupation campaniforme. In H. Dartevelle (ed.), Actes du Colloque de ClermontFerrand "V Vencontres méridionales de la Préhistoire récente ». Préhistoire du Sud-Ouest, Cressensac, 493-507.

DARRACQ S., GODRON M. \& ROMANE F., 1984 - Typologie Forestière de la région des Garrigues du Gard. ENGREF, Nancy, $181 \mathrm{p}$.

FABRE G. \& MONTEIL M., 2001 - Sur l'hydrogéomorphologie d'un espace à forte anthropisation urbaine : le site de Nîmes (Languedoc, France) du Pléistocène supérieur à l'Antiquité ; impacts postérieurs. Comptes Rendus de l'Académie des Sciences de Paris. Sciences de la Terre et des planètes, 333, 435-440.

FREITAS (DE) L., CHARLES V., HAMEAU P., JALLOT L., PAHIN A.-C., SÉNÉPART I. \& VEYSSIERE F., 1987 - Le Moulin Villard, Caissargues - Gard. Document final de synthèse, SRA Languedoc-Roussillon, INRAP, rapport non publié, Montpellier.

FREITAS (DE) L., CHARLES V., ESCALLON G., JALLOT L. \& SÉNÉPART I., 1988 - Le Moulin Villard, Caissargues - Gard. Document final de synthèse, SRA Languedoc-Roussillon, INRAP, rapport non publié, Montpellier.

GARMY P. \& MONTEIL M., 2000 - Le quartier antique des Bénédictins à Nîmes (Gard). Découvertes anciennes et fouilles 19661992. Documents d'Archéologie Française, 81, Maison des Sciences de l'Homme, Paris, 280 p.

GOEURY C., 2000 - Gpalwin for Windows 95/98/NT, version 990421. IMEP, Marseille.

GUTHERZ X. \& JALLOT L., 1994 - Approche géoculturelle des pays fontbuxiens. In J. Vaquer (ed.), Le Néolithique du Nord-ouest méditerranéen. Actes du $24^{\mathrm{e}}$ Congrès préhistorique de France, septembre 1994, Société Préhistorique Française, Paris, 161-174.

HEINZ C. \& THIÉBAULT S., 1998 - Characterization and Paleoecological Significance of Archaeological Charcoal Assemblages during Late and Post-Glacial Phases in Southern France. Quaternary Research, 50, 58-68.

HERVÉ M.-L., 2000a - Nîmes (30), ZAC de Haute Magaille. Document final de synthèse, SRA Languedoc-Roussillon, INRAP, rapport non publié, Montpellier, $78 \mathrm{p}$.

HERVÉ M.-L., 2000b - Fossé de Haute-Magaille. Document final de synthèse, SRA Languedoc-Roussillon, INRAP, rapport non publié, Montpellier, $79 \mathrm{p}$.

JALLOT L. (ed.), 2005 - Mas de Vignoles IV, à Nîmes (Gard). Document final de synthèse, SRA Languedoc-Roussillon, INRAP, rapport non publié, Montpellier.

JORDA M. \& PROVANSAL M., 1996 - Impact de l'anthropisation et du climat sur le détritisme en France du sud-est (Alpes du sud et Provence). Bulletin de la Société Géologique de France, 167 (1), $159-168$. 
JUNG C., CHEVILLOT P., JORDA C., LABARUSSIAT C. \& RAYNAUD F., 2002 - Ligne à Grande Vitesse - Contournement ferroviaire de Nîmes à Montpellier. Document final de synthèse, SRA Languedoc-Roussillon, INRAP, rapport non publié, Montpellier, $120 \mathrm{p}$

KERNEY M. P. \& CAMERON R. A. D., 1999 - Guide des escargots et limaces d'Europe. Delachaux et Niestlé, Paris, $370 \mathrm{p}$

LABAUNE C., 2001 - L'invasion de la région méditerranéenne française par l'escargot Xeropicta derbentina: mécanismes, conséquences écologiques et agronomiques. Thèse de doctorat, Université Aix-Marseille III, 162 p.

LABAUNE C. \& MAGNIN F., 2002 - Pastoral management vs. land abandonment in Mediterranean uplands: impact on land snails communities. Global Ecology \& Biogeography, 11, 237-245.

LEMERCIER O., 1998 - Phénomène, culture et tradition : statuts et rôles du Campaniforme au $\mathrm{III}^{\mathrm{e}}$ millénaire dans le Sud-Est de la France. Bulletin de la Société Préhistorique Française, 95 (3), 365-382.

LEMERCIER O., 2000 - Espace culturel, territoire et terroir : approches spatiales des groupes campaniformes récents dans le sudest de la France. In M. Leduc, N. Valdeyron \& J. Vaquer (eds.), $I V^{e}$ Rencontres Méridionales de Préhistoire récente «Temps et Espaces Culturels », Troisième session, Toulouse, 1998. Archives d'Écologie Préhistorique, Nîmes, 177-186.

MAGNIN F., 1991 - Mollusques continentaux et histoire quaternaire des milieux méditerranéens (Sud-Est de la France, Catalogne). Thèse de doctorat, Université Aix-Marseille II, 364 p.

MAGNIN F. \& TATONI T., 1995 - Secondary successions on abandoned cultivation terraces in calcareous Provence. II - The gastropod communities. Acta Oecologica, 16 (1), 89-101.

MAGNIN F., TATONI T., ROCHE P. \& BAUDRY J., 1995 - Gastropod communities, vegetation dynamics and landscape changes along an old-field succession in Provence, France. Landscape and Urban Planning, 31, 249-257.

MAGNY M., 1995 - Une histoire du climat : des derniers mammouths au siècle de l'automobile. Errance, Paris, $176 \mathrm{p}$.

MARTIN S. \& MAGNIN F., 2002 - Caractérisation des perturbations anthropiques dans la séquence holocène de l'Ubac (Goult, Vaucluse), à partir de la malacofaune. In $\mathrm{H}$. Richard \& A. Vigno (eds.), Équilibres et ruptures dans les écosystèmes depuis 20000 ans en Europe de l'Ouest, Actes du colloque international de Besançon, septembre 2000, Presses Universitaires Franc-Comtoises, Besançon, 205-212.

MARTIN S., MAGNIN F. \& KISS L., 2003 - Land snails and human impact: the temporal resolution of Holocene assemblages. In É Fouache (ed.), The Mediterranean World Environment and History. Elsevier SAS, Paris, 235-246.

MARTIN S., 2004 - Caractérisation de l'anthropisation à l'Holocène en Provence et en Languedoc oriental, par les mollusques terrestres. Thèse de doctorat, Université Paris 1 - Panthéon-Sorbonne, $429 \mathrm{p}$.

MARTIN S. \& MAGNIN F., sous-presse - Gastéropodes terrestres et anthropisation : peut-on distinguer le signal anthropique du signal climatique à l'Holocène ? Exemples du domaine méditerranéen français. In Actes de la Table Ronde en l'honneur de R. Neboit-Guilhot, "L'érosion entre société, climat et paléoenvironnement », Clermont-Ferrand, 25-27 mars 2004, Presses Universitaires Blaise Pascal, collection « Nature et Sociétés ».
MONTEIL M., (ed.), 1993 - Les fouilles de la ZAC des Halles à $N \hat{\imath}-$ mes (Gard). Bulletin de l'École Antique de Nîmes, Supplément 1, $322 \mathrm{p}$.

MONTEIL M., 1999 - Nîmes antique et sa proche campagne. Étude de topographie urbaine et périurbaine (fin $\mathrm{VI}^{e}$ s. av. J.-C. / VI $I^{e}$. ap. J.-C.). Monographies d'Archéologie Méditerranéenne, 3, Lattes, $528 \mathrm{p}$.

PISKORZ M., AURAND J.-L., BIOUL C., CHEVILlOT P., LANCELOT S. \& RECOLIN A., 2002 - Mas des Abeilles I t II.4 à Nîmes (Gard), Document final de synthèse, SRA LanguedocRoussillon, INRAP, rapport non publié, Montpellier, 63 p.

PFENNINGER M., 2002 - Relationship between microspatial population genetic structure and habitat heterogeneity in Pomatias elegans (O.F. Müller 1774) (Caenogastropa, Pomatiasidae). Biological Journal of the Linnean Society, 76, 565-575.

POMARÈDES H. \& RASCALOU P., 2002 - ZAC de Vignole, Bassin Sud, Tranche 2 à Nîmes (Gard), Genèse et évolution de l'habitat et organisation de l'espace rural à "Vignole VII / Gouffre des Bouchers », Document final de synthèse, SRA Languedoc-Roussillon, INRAP, rapport non publié, Montpellier, $107 \mathrm{p}$.

PUERTAS O., 1998 - Palynologie dans le delta du Lez. Contribution à l'histoire du paysage de Lattes. Édition de l'Association pour la Recherche Archéologique en Languedoc Oriental, Lattes, 181 p.

ROUSSEAU D.-D., 1985 - Structures des populations quaternaires de Pupilla muscorum (gastropode) en Europe du Nord. Relations avec leurs environnements. Thèse de doctorat, Université de Dijon, $133 \mathrm{p}$.

SEJALON P., CHEVILlOT P., MANEN C., MARTIN S., PERRIN T., AUDOUIT F., LANCELOT S. \& LELIEVRE V., 2004 - Mas de Vignoles VI à Nîmes (Gard), Document final de synthèse, SRA Languedoc-Roussillon, INRAP, rapport non publié, Montpellier, $79 \mathrm{p}$.

SPARKS B. W., 1961 - The ecological interpretation of quaternary non-marine mollusca. Proceedings Linnean Society of London, 172, 71-80.

THIEBAULT S., 1995 - Dégradation et/ou substitution du milieu végétal au Néolithique en Provence. In S. Van der Leeuw (ed.), L'homme et la dégradation de l'environnement. $\mathrm{XV}^{\mathrm{e}}$ Rencontres Internationales d'Archéologie et d'Histoire d'Antibes, Éditions APDCA, Juan-les-Pins, 185-194.

TRIAT-LAVAL H., 1978 - Contribution pollenanalytique à l'histoire Tardi-\&Postglaciaire de la végétation de la basse vallée du Rhône. Thèse de Doctorat, Université Aix-Marseille III, 343 p.

VERNET J.-L. \& THIÉBAULT S., 1987 - An approach to northwestern Mediterranean recent prehistoric vegetation and ecologic implications. Journal of Biogeography, 14, 117-127.

VERNET J.-L., 1991 - L'histoire du milieu méditerranéen humanisé révélée par les charbons de bois. In J. Guilaine (ed.), Pour une archéologie agraire. Armand Colin, Paris, 369-408.

VERNET J.-L., 1997 - L'homme et la forêt méditerranéenne, de la Préhistoire à nos jours. Errance, Paris, $248 \mathrm{p}$.

WAINWRIGHT J., 2000 - Contextes géomorphologiques et géoarchéologiques des habitats de l'Âge du Bronze en Méditerranée occidentale. In J. Gasco \& F. Claustre (eds.), Habitats, économies et sociétés du Nord-Ouest méditerranéen de l'Âge du Bronze au premier Âge du Fer, Actes du XXIV ${ }^{\mathrm{e}}$ Congrès Préhistorique de France, Carcassonne 26-30 septembre 1994, 11-26. 